\title{
Statistical Characterization of Process Variables on the Operation of Continuous Tracked Vehicles
}

\author{
Jorge Duarte Forero ${ }^{1 *}$, Guillermo E. Valencia' and Luis G. Obregon ${ }^{2}$ \\ 'Department of Mechanical Engineering, KAI Research Group, Universidad del Atlantico, Barranquilla, Colombia; \\ jorgeduarte@mail.uniatlantico.edu.co,guillermoevalencia@mail.uniatlantico.edu.co \\ 2Department of Chemical Engineering, Sustainable Chemical and Biochemical Processes Research Group, Universi- \\ dad del Atlantico, Barranquilla, Colombia; luisobregon@mail.uniatlantico.edu.co
}

\begin{abstract}
Background/Objectives: The implementation of Design of Experiments as a tool for the study of process variables related to the operation of some types of industrial machinery, such as Continuous Tracked Vehicles, as a base for optimization processes that allow establishing an operational point with the most efficient use of these vehicles. Methods: Using a factorial design, a series of experiments were realized, with some process variables as design factors, each one arranged with two design levels. From these, statistical methods were used to validate the normal distribution of the results and at the same time, to establish mathematical expressions that correlate said parameters. Findings: The results allowed the determination of the factors that can be neglected in optimization processes, due to their low influence of the operation characteristics; besides, it was demonstrated that certain parameters, as the terrain, have little or no influence on the vehicle performance, which serves as a valid criterion to evaluate future investments inload transport systems. Application: To develop a tool for the prediction of the operation characteristics of Continuous Tracked Vehicles which will be used for the estimation of potential savings generated by more efficient use of these machines?
\end{abstract}

Keywords: Continuous Tracked Vehicles, Design of Experiments, Operation Characteristics, Performance, Statistical Model

\section{Introduction}

Continuous Tracked Vehicles (from now on referred as CTV, by their initials) are vehicles driven by a continuous band of threads or track plates, which are put in motion using two or more wheels. As opposed to the conventional propulsion systems, such as rubber or metal wheels, the large surface area of the tracks enable these type of vehicles to traverse soft ground with less likelihood of becoming stuck due to sinking, thus making these suitable for industrial applications where it is required to carry heavy loads across unstable and wet terrain, such as excavators and tractors, in which the conventional propulsion systems are unable to work properly ${ }^{1-3}$ torque transducer, National Instrument cRIO-9004 CompactRIO Real-time Controller Unit (RCU.
Even with the good points of CTV, there's still a need to solve: to achieve higher efficiencies in the use of these vehicles; their operating characteristics have to be improved; however, this is heavily dependent of the environmental and working conditions ${ }^{4}$. Besides this fact, the relationship between these parameters and the operation characteristics is not easy to calculate, because some factors are of qualitative nature, which makes difficult the direct formulation of a mathematical model using a thorough analysis of the system, and therefore the estimation of an optimum point of operation with the highest performance $\mathrm{e}^{5}$.

In order to overcome these situations in which qualitative and quantitative factors are involved, statistical methods were developed in order to estimate mathematical models that take into account the interaction of all

*Author for correspondence 
these factors over a specific response; from these methods, the Design of Experiments (abbreviated as DOE), is the most common in the development of said models ${ }^{6}$. Though DOE has its own limitations, such as the generated model only applies to the system in the studied conditions, its prediction accuracy makes it a powerful tool to study complex cases when a mathematical analysis isn't enough to correlate all the studied parameters with the response required $\mathrm{d}^{\mathrm{7-9}}$.

As said before, the operation characteristics in CTV is related to qualitative and quantitative factors associated with the work conditions. However, which factors influence the operation of these vehicles? To solve this question, it is required to evaluate the influence of each variable over an operation characteristic of interest, such the required time for the displacement of a CTV, to apply statistical methods that can define on a solid basis, if a factor is relevant over the output variable. In order to do so, a methodology based on Design of Experiments is applied to study a set of 4 variables and their influence over the required time, to establish if these variables have an influence over the displacement speed, according to some statistical criteria; these results can be used as base for future works, oriented to the modelling and optimization of the operation characteristics of CTV, discarding the variables that don't affect the output of these vehicles.

\section{Methodology}

This section presents a detailed description of the general purpose of the study developed, the presentation of the aspects of the experimental configuration used and finally the fundamental theory behind the Design of Experiments.

\subsection{Purpose and Description of the Study}

The main purpose of the study developed is to analyze the influence of certain parameters, such as the terrain, engine usage, vehicle controls usage and engine angular speed, over operation characteristics of the vehicle, mainly the displacement time of a CTV; in order to do so, a $2^{\mathrm{k}}$ factorial design was selected to create the design matrix and to define the level combinations of each factor required in the evaluation of the results. These were measured in the experimental conditions and given the fact statistical analyses usually require a huge amount of time to do all the calculations required, a computer package was used in the processing of the studied data. By its speed and processing capabilities, the commercial software Minitab was used. Within the said package, graphical and statistical methods, such as the Analysis of Variance (ANOVA) were applied to analyze the influence of each factor, as well as their interaction, over the measured variable and therefore verify their level of significance, for a specific degree of reliability.

\subsection{Experimental Equipment}

For the experiment, a Komatsu excavator, used in applications of waste management, was selected. The equipment has between 6250 and $6750 \mathrm{Hr}$ of work, for which they are in conditions of similar use and wear. A test track of 15 meters in concrete and 1 of sand/clay with the same length was available for the execution of the sequences of the randomized tests. To avoid the influence of undesired factors, the runs are executed with the hot equipment (with hydraulic oil at $70^{\circ} \mathrm{C}$ ), which is the steady state of operation of this fluid (by the thermostats of the hydraulic system, which open at this value), since it remains fixed at this value and the process is not influenced by the viscosity of the oil and the decrease of it.

\subsection{Fundamental Theory and Equations}

To improve the efficiency and performance of industrial processes, it is necessary to establish a fundamental equation that allows correlating an output of interest and the factors that influence it. Using the factors $\mathrm{x}_{1}, \mathrm{x}_{2} \ldots, \mathrm{x}_{\mathrm{n}}$ and the output $y$, a process can be described or modeled as shown in the Equation 1:

$$
\mathrm{y}=\mathrm{f}\left(\mathrm{x}_{1}, \mathrm{x}_{2}, \ldots, \mathrm{x}_{\mathrm{n}}\right)
$$

Where is stated that these factors would affect the desired output. However, equation one cannot be used in practical applications (it is only a mathematical formulation). To worse things, many processes are dependent on a huge number of factors, which makes process modeling a hard operation. Given this, a question is raised: Is there a way to decrease the number of factors involved? The former question is related to the possibility to reduce the Equation 1 into another with less independent variables, such as Equation 2;

$$
y=f\left(x_{1}, x_{2}\right)
$$


Where only the influent factors remain in the equation; with these, a formal expression can be derived, based in a linear combination of single and combined factors, such as the Equation 3:

$$
\mathrm{y}=\mathrm{a}+\mathrm{b} \cdot \mathrm{x}_{1}+\mathrm{c} \cdot \mathrm{x}_{2}+\mathrm{d} \cdot \mathrm{x}_{1} \cdot \mathrm{x}_{2}
$$

This reduction has a great influence over the modeling of any system, allowing to obtain a process equation that is easier to operate and optimize, with a little loss of accuracy. However, in reality, some points needed to be solved before doing this process: For example, any processes and operations use factors of qualitative nature, which are harder to include into a model than these of quantitative nature; besides that, it is required to establish a solid criteria to separate the factors by their influence, which is commonly measured at certain experimental values.

As a solution of the previous issues, the Design of Experiments was established as a method to statistically define, from experimental data, whether a factor is relevant or not in the model development, based in probability distributions and such. The main basis for DOE is the generation of a set of measurement points, based on the knowledge of three basic parameters of the design $\frac{6,10,11}{\text { : }}$

- The number of controllable factors to be evaluated.

- The number of levels for each factor, that is, the number of values that each factor can take.

- The number of replicas of the design, which correspond to the number of repetitions that will be executed.

The measurement points originated, form the design matrix, which contains the list of all combinations or treatments that can be formed considering all possible combinations of factor levels; for example, when two factors $\mathrm{x}_{1}$ and $\mathrm{x}_{2}$ are used, with two levels each (that is, a 2 $\mathrm{x} 2$ design), 4 possible combinations are obtained in the design matrix, illustrated in Figure 1. Due to this structure, this kind of DOE is called Factorial Design $2^{\mathrm{k}}$, where $\mathrm{k}$ indicates the number of factors involved in the design.

Following the design definition, experimental data is measured at each combination of factor levels; however, the analysis of this results require the establishment of evaluation methods, to generate a solid base for evaluation and comparison of the influence generated by each factor. From these methods, the most used is the Analysis of Variances (or ANOVA, by ANalysis Of VAriance), which uses dispersion measures to determine if the variability of a given factor is influential on the total variability of the output of the system, under the assumption that the data obey a normal distribution of probability; however, it is widely used to analyze non-normal distributions with variances between relatively low factors. From the variances of the factors and the output, the ANOVA calculates for each factor a test statistic $\mathrm{F}$, which is compared with Fisher's probability distribution $\mathrm{F}$ (commonly used in hypothesis testing), to obtain the so-called P-value, which defines a factor studied as statistically relevant or not (for a given reliability, usually $95 \%$ 을 by comparing with the critical P-value, as in the Equation 4:

$$
\mathrm{P}\left(\mathrm{F}_{\text {data }}\right) \leq \mathrm{P}\left(\mathrm{F}_{\text {critical }}\right)
$$

Where the critical P-value is defined by the specified reliability of the analysis. In addition to the above, the results of the ANOVA analysis can be organized into a series of graphical tools, which facilitate their understanding; the most used are the following:

- The graphs of main effects, which allows knowing initially if each factor studied individually influences the response, from the behavior of the latter when only one factor is varied at a time; in this method, it is considered that a factor has no influence, when the variation in the output is minimal or null between levels of the factor (as shown in Figure 2a, while the highly influential factors are characterized by generating considerable changes in the output (which is shown in Figure 2b) and the severity of the changes, qualitatively determines the influence of this factor.

- The interaction graphs, which are a useful tool when it is necessary to visualize the influence of the interaction of said factors on the output of a system or process. As shown in Figure 3, this type of graph evaluates the evolution of the response with respect to one factor, for different levels of a second factor and determines that there is an interaction, when these curves are not parallel and the intensity of their influence is determined qualitatively by the degree of parallelism between the curves.

- The Pareto diagram, which organizes the effects according to their standardized impact on the response variable, from the values calculated by the ANOVA analysis, as shown in Figure 4. 


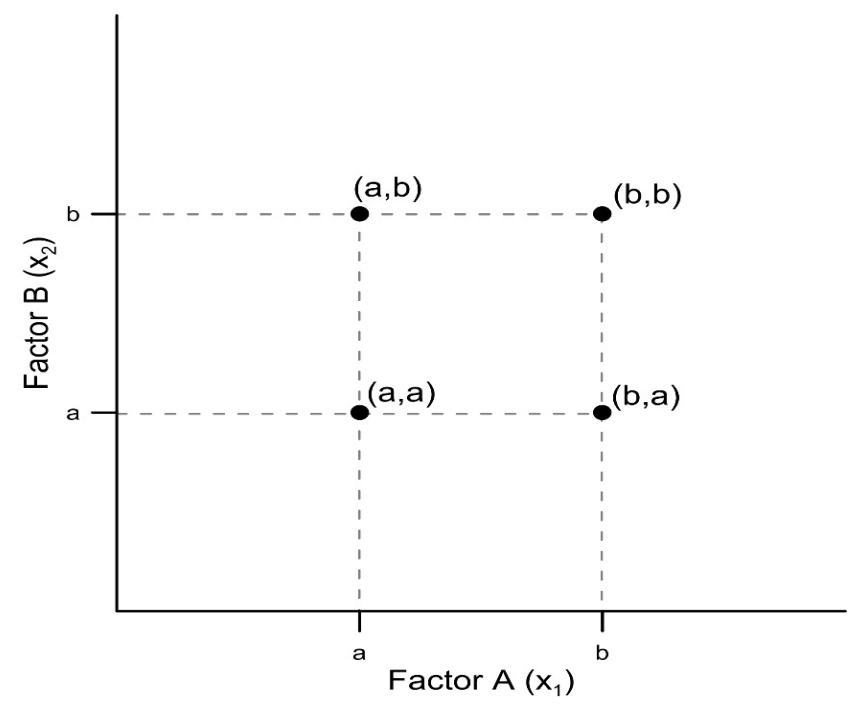

Figure 1. Design matrix for a design $2 \times 2$.

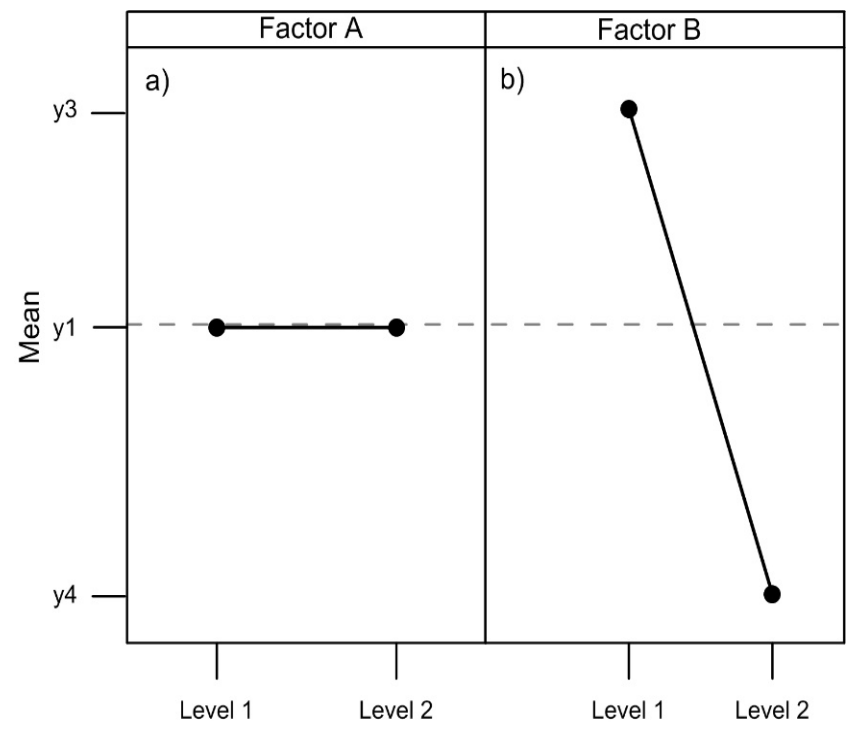

Figure 2. Schematic of a main effects plot.

\section{Result and Discussion}

As said before, the objective of the study was to establish the factors (or combinations of factors) that notably influence the operation characteristic evaluated (displacement time). To do so, a factorial design $2^{4}$ was used, with two replicas, in order to achieve better accuracy in the results; to minimize variations due to external factors, the design matrix was randomized and the results were processed in the Minitab package, using a name convention for the factors: Factor A (terrain), Factor B (engine usage), Factor $\mathrm{C}$ (controls usage) and Factor D (engine rpm). Finally, the results were expressed graphically to give an easy understanding of these.

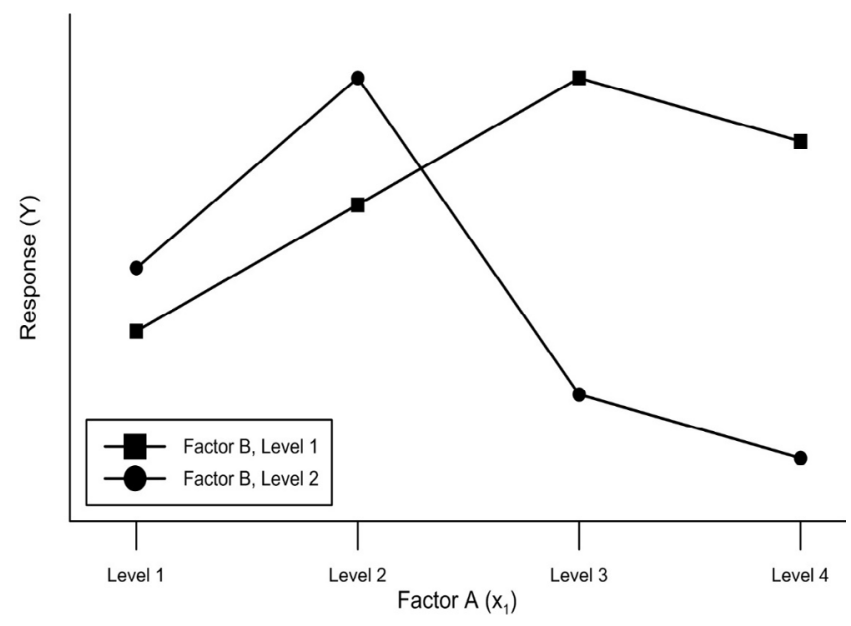

Figure 3. Schematic of an interaction plot.

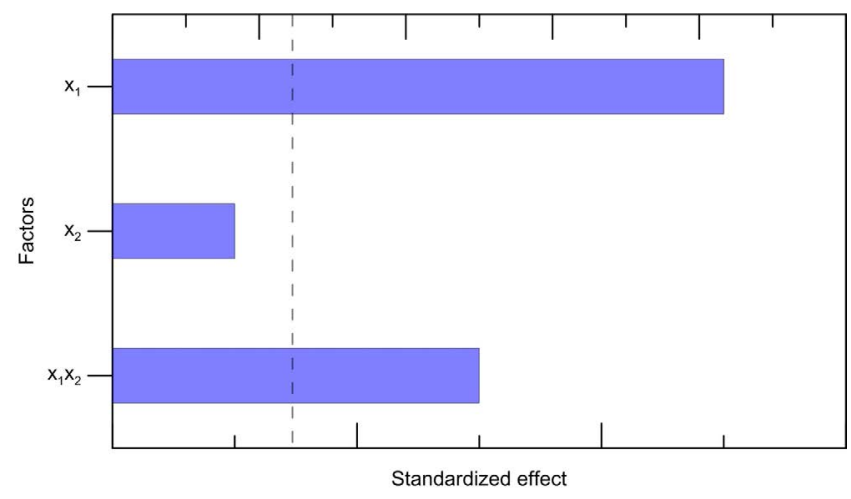

Figure 4. Pareto diagram of standardized effects.

\subsection{Study of the Influence of Main Factors}

Figure 5 shows the main effects plot for each factor; as shown, plots for factors A and B have small slopes, when these are switched from a level to another. According to the previous theory, low slopes mean that these factors have little to no effect over the system response and therefore can be neglected in a process modeling. An interesting point of Figure 5, resides in the fact Factor A is the terrain: though common sense would expect terrain having a huge influence over the displacement time, it isn't true in the real case; due to this, investments done to improve the quality of the terrain at the work site are not required, which represents a long-term money saving and a more efficient usage of industrial resources. Though the influence of factors A and B over the system varies from little to none, factors $\mathrm{C}$ and $\mathrm{D}$ have a more pronouncedeffect over it, which can be seen in the high slope shown in Figure 5; therefore, a model for the displacement time would take into account the latter factors in the modelling of the desired output. 


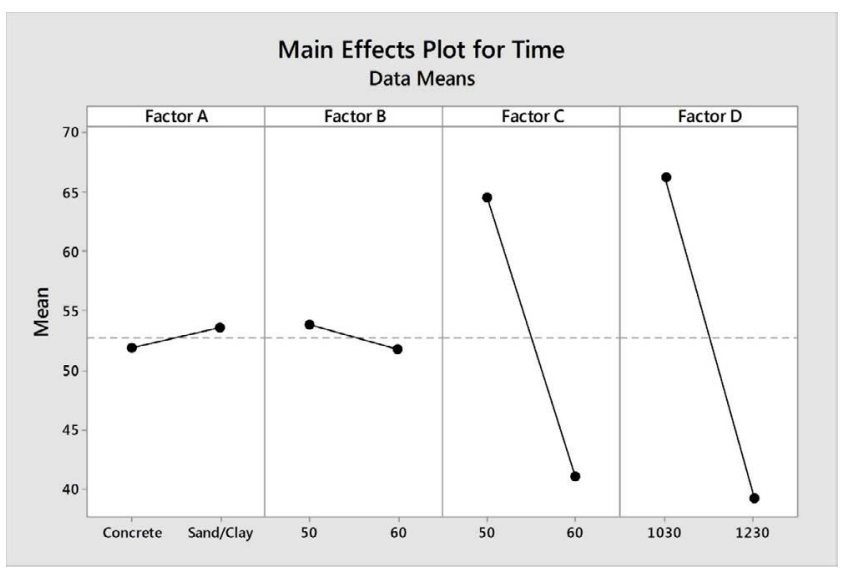

Figure 5. Main effects plotted in Minitab.

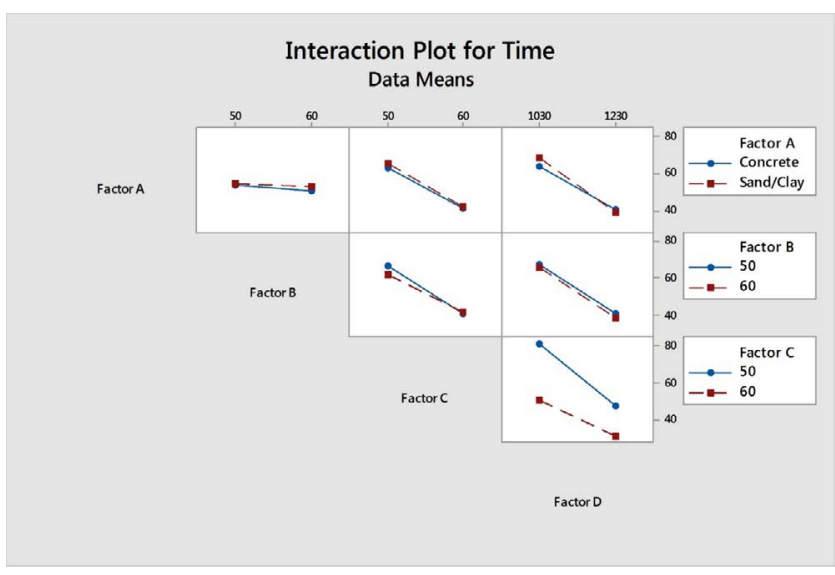

Figure 6. Interaction graph plotted in Minitab.

\subsection{Study of the Influence of the Factor}

\section{Interactions}

Once the influence of main effects is evaluated, the next step in the analysis involved the estimation of the changes in the behavior in the output variable (displacement time) when two or more factors are modified at the same time. Keeping this in mind, Figure 6 shows the interaction plots for each possible combination formulated; from these, it can be found that from all the possible interactions, the only that have an influence on the output, is the combination between factors $C$ and D, while all the others do not generate a notable change in it. Though the interaction plots allow establishing this relationship between factor combinations and output, this only can be defined in a qualitative way using this method; however, the Pareto diagram, shown in Figure 7, allows reassuring this relationship in a quantitative form, by calculating the test statistic $\mathrm{F}$ for each one of these. By comparison with a threshold value (which varies with reliability), it was demonstrated that, based on the experimental data, the displacement time of a CTV, is heavily influenced by the following:

- The controls' percentage of usage (Factor C).

- The engine speed in RPM (Factor D).

- The interaction between said factors (Interaction CD).

\subsection{Validation of the Main Assumption}

As said before, the Analysis of Variance is commonly used when the output variable behaves according to a normal distribution of probability. Therefore, to validate the results previously shown, it is required to validate the main assumption of the ANOVA itself and to do so, the normal distribution plot for the time values measured in the experimental setup was plotted in the Figures 8 and 9, where it is also plotted the significative factors $(C, D, C D)$. From this graph, can be seen that the data adjusts to the normal distribution, with a certain percentage of reliability (95\% in this case) and this fact allows to validate the results previously shown, as these go in concordance with the theory previously explained and the qualitative findings were done by the graphical methods (main effects and interaction plots).

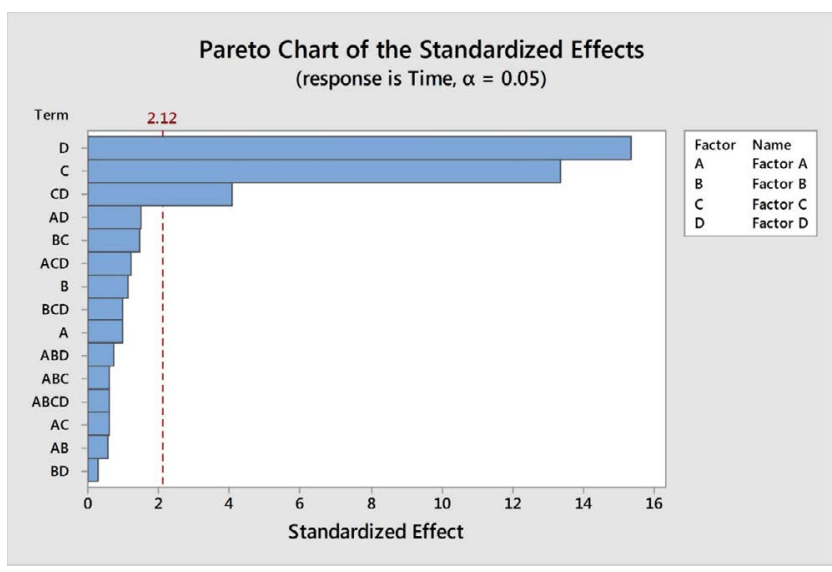

Figure 7. Pareto of standardized effects for the studied case. 


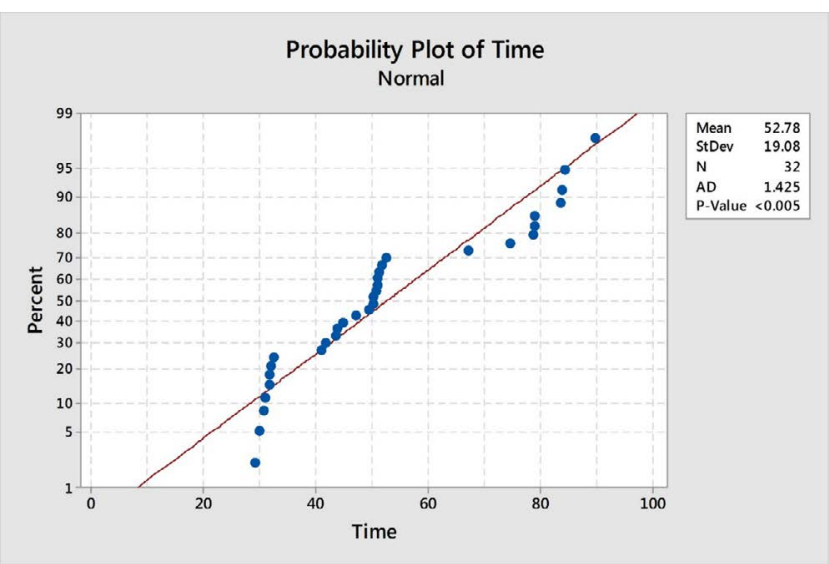

Figure 8. Normal probability plot.

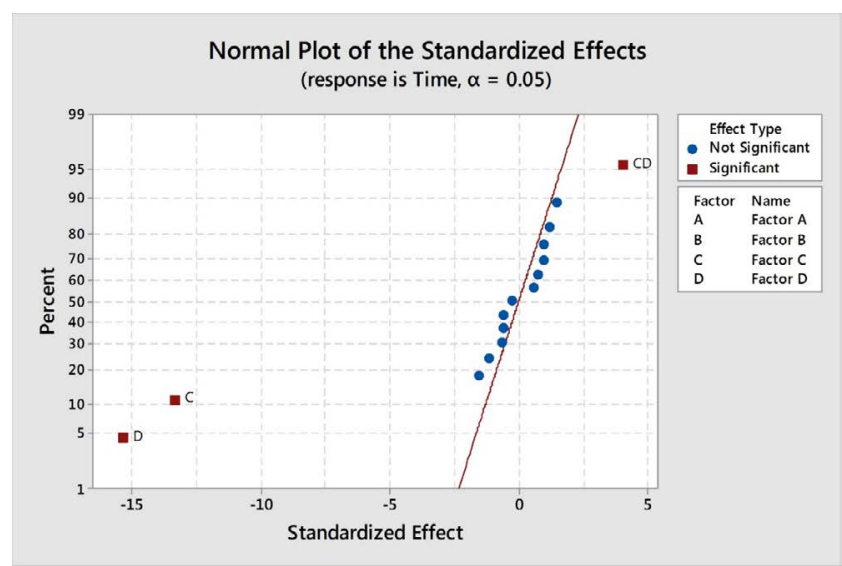

Figure 9. Normal probability plot and influencing effects.

\section{Conclusions}

From the present study, a certain set conclusion was established: In the first place, the application of DOE allowed to establish the factors that influence the variable studied and also the factors that can be neglected from modelling and optimization methods, given the fact their standardized effect are not enough to keep these into account. By doing that, DOE and particularly factorial design, proved to be useful as a tool to study and evaluate engineering processes and applications where non-quantitative data is involved and given the advantages of the analysis ANOVA in the evaluation of a wide type of probability distributions, these two tools are used in almost every field of engineering and scientific investigation. Finally, the present work determined the engine speed and control usage as the most influencing variables, as same as their interaction, neglecting the little to no variation other factors generate (as it was shown in the corresponding graphs) which discards future investments in non-rele- vant factors, such as the terrain; however, to carry formal optimization processes, it is required to establish mathematical expressions to correlate and predict accurately the response outside the experimental data, which corresponds to the application of regression models, which will be applied to the case of study in a later work.

\section{Acknowledgment}

Acknowledgments for the support provided by Universidad del Atlantico, Barranquilla - Colombia through of Efficient Energy Management Research Group Kai, with J. Duarte - G. Valencia and Sustainable Chemical and Biochemical Processes Research Group, with L. Obregon.

\section{References}

1. Rahman A, Yahya A. Performance investigation of an advanced tracked prime mover on the low bearing soil. Journal of Terramechanics. 2013; 50(4):233-44. https://doi. org/10.1016/j.jterra.2013.06.001

2. Okada Y, Nagatani K, Yoshida K, Yoshida T, Koyanagi E. Shared autonomy system for turning tracked vehicles on rough terrain using real-time terrain scanning. 2010 International Conference on Advanced Mechatronics. 2010; 5:357-62. https://doi.org/10.1299/jsmeicam.2010.5.195

3. Negrut N, Melanz D, Mazhar H, Lamb D, Jayakumar P, Letherwood $M$. Investigating through simulation the mobility of light tracked vehicles operating on discrete granular terrain. SAE International Journal of Passenger Cars - Mechanical Systems - V122-6EJ. SAE International Journal of Passenger Cars - Mechanical Systems-V122-6. 2013; 6(1):369-81.

4. Okada Y, Nagatani K, Yoshida K, Tadokoro S, Yoshida T, Koyanagi E. Shared autonomy system for tracked vehicles on rough terrain based on continuous three-dimensional terrain scanning. Journal of Field Robotics. 2011; 28(6):875-93. https://doi.org/10.1002/rob.20416

5. Tiwari VK, Pandey KP, Pranav PK. A review on traction prediction equations. Journal of Terramechanics. 2010; 47(3):191-9. https://doi.org/10.1016/j.jterra.2009.10.002

6. Triola M. Estadística. 10th Ed. Mexico: Pearson Educación de Mexico, S.A. 2009. p. 1-914.

7. Juang LW, Kollmeyer PJ, Anders AE, Jahns TM, Lorenz $\mathrm{RD}$, Gao D. Investigation of the influence of superimposed AC current on lithium-ion battery aging using statistical Design of Experiments. Journal of Energy Storage. 2017; 11:93-103. https://doi.org/10.1016/j.est.2017.02.005 
8. Garcia S, Fernandez A, Luengo J, Herrera F. Advanced nonparametric tests for multiple comparisons in the Design of Experiments in computational intelligence and data mining: Experimental analysis of power. Information Sciences. 2010; 180(10):2044-64. https://doi.org/10.1016/j.ins.2009.12.010

9. Naus G, Vugts R, Ploeg J, van de Molengraft R, Steinbuch M. Cooperative adaptive cruise control, design and experiments. IEEE Proceedings of the American Control
Conference; 2010. p. 6145-50. https://doi.org/10.1109/ ACC.2010.5531596

10. Vicente G, Coteron A, Martinez M, Aracil J. Application of the factorial Design of Experiments and response surface methodology to optimize biodiesel production. Industrial Crops and Products. 1998; 8(1):29-35. https:// doi.org/10.1016/S0926-6690(97)10003-6

11. Hernandez R, Fernandez C, Baptista M del P. Metodología de la investigacion. 5th Ed. Mexico D.F.: McGraw-Hill; 2010 . 